This item was submitted to Loughborough's Research Repository by the author.

Items in Figshare are protected by copyright, with all rights reserved, unless otherwise indicated.

\title{
Modelling approaches to the dewetting of evaporating thin films of nanoparticle suspensions
}

PLEASE CITE THE PUBLISHED VERSION

http://dx.doi.org/10.1088/0953-8984/21/26/264016

PUBLISHER

(c) IOP Publishing Ltd

\section{VERSION}

AM (Accepted Manuscript)

\section{PUBLISHER STATEMENT}

This work is made available according to the conditions of the Creative Commons Attribution-NonCommercialNoDerivatives 4.0 International (CC BY-NC-ND 4.0) licence. Full details of this licence are available at: https://creativecommons.org/licenses/by-nc-nd/4.0/

\section{LICENCE}

CC BY-NC-ND 4.0

\section{REPOSITORY RECORD}

Thiele, Uwe, loan Vancea, Andrew J. Archer, Mark J. Robbins, Lubor Frastia, A. Stannard, E. Pauliac-Vaujour, C.P. Martin, M.O. Blunt, and P.J. Moriarty. 2019. "Modelling Approaches to the Dewetting of Evaporating Thin Films of Nanoparticle Suspensions". figshare. https://hdl.handle.net/2134/16005. 


\title{
Modelling approaches to the dewetting of evaporating thin films of nanoparticle suspensions
}

\author{
U. Thiele*, I. Vancea, A. J. Archer, M. J. Robbins, L. Frastia \\ Department of Mathematical Sciences, \\ Loughborough University, Leicestershire LE11 3TU, UK
}

\author{
A. Stannard, E. Pauliac-Vaujour, C. P. Martin, M. O. Blunt, P. J. Moriarty \\ The School of Physics and Astronomy, \\ The University of Nottingham, Nottingham NG7 2RD, UK
}

\footnotetext{
* homepage: http://www.uwethiele.de, u.thiele @lboro.ac.uk
} 


\begin{abstract}
We review recent experiments on dewetting thin films of evaporating colloidal nanoparticle suspensions (nanofluids) and discuss several theoretical approaches to describe the ongoing processes including coupled transport and phase changes. These approaches range from microscopic discrete stochastic theories to mesoscopic continuous deterministic descriptions. In particular, we focus on (i) a microscopic kinetic Monte Carlo model, (ii) a dynamical density functional theory and (iii) a hydrodynamic thin film model.

Models (i) and (ii) are employed to discuss the formation of polygonal networks, spinodal and branched structures resulting from the dewetting of an ultrathin 'postcursor film' that remains behind a mesoscopic dewetting front. We highlight, in particular, the presence of a transverse instability in the evaporative dewetting front which results in highly branched fingering structures. The subtle interplay of decomposition in the film and contact line motion is discussed.

Finally, we discuss a simple thin film model (iii) of the hydrodynamics on the mesoscale. We employ coupled evolution equations for the film thickness profile and mean particle concentration. The model is used to discuss the self-pinning and de-pinning of a contact line related to the 'coffee-stain' effect.

In the course of the review we discuss the advantages and limitations of the different theories, as well as possible future developments and extensions.

The paper is published in: J. Phys.-Cond. Mat. 21, 264016 (2009), in the Volume "Nanofluids on solid substrates" and can be obtained at http://dx.doi.org/10.1088/0953-8984/21/26/264016
\end{abstract}




\section{INTRODUCTION}

The patterns formed in dewetting processes have attracted strong interest since Reiter analysed the process quantitatively in the early nineties. In these experiments, that proved to be a paradigm in our understanding of dewetting, a uniform thin film of polystyrene (tens of nanometers thick) is deposited on a flat silicon oxide substrate is brought above the glass transition temperature. The film ruptures in several places, forming holes which subsequently grow, competing for space. As a result, a random polygonal network of liquid rims emerges. The rims may further decay into lines of small drops due to a Rayleigh-type instability [1-3]. The related problems of retracting contact

lines on partially wetting substrates and the opening of single holes in rather thick films have also been studied [4, 5].

Subsequent work has mainly focused on many different aspects of the dewetting process for simple non-volatile liquids and polymers (for reviews see Refs. [6-8]). All stages of the dewetting of a film are studied: the initial film rupture via nucleation or a surface instability (called spinodal dewetting) [1, 9-13], the growth process of individual holes [14-16], the evolution of the resulting hole pattern [3, 13], and the stability of the individual dewetting fronts [17-[19]. We note in passing, that descriptions of dewetting patterns may also be found in historic papers, particularly for the dewetting of a liquid film on a liquid substrate. Tomlinson [20, footnote 18 on p. 40] considered turpentine on water and Marangoni [21, p. 352f] oil on water.

More recently, interest has turned to the dewetting processes of solutions and suspensions. However, these systems have not yet been investigated in any great depth. Such systems are complicated because their behaviour is determined by the interplay between the various solute (or colloid) and solvent transport processes. Furthermore, the solvents that are used often evaporate, i.e., one has to distinguish between 'normal' convective dewetting and evaporative dewetting. A number of experiments have been performed employing (colloidal) solutions of polymers [22-25], macromolecules like collagen and DNA [26-31] and nanoparticles [32-40]. The latter are sometimes referred to as 'nanofluids'. The initial focus of much of the research in the field has been on investigating the structures that are formed which are similar to the ones observed in the "classical' dewetting of non-volatile liquids. Labyrinthine structures and polygonal networks result from spinodal dewetting and heterogeneous nucleation and growth, respectively. They are 'decorated' with the solute and therefore conserve the transient dewetting pattern as a dried-in structure when all the solvent has evaporated [28, 34]. The picture is, however, not complete. The solute may 
also shift the spinodal and binodal lines as compared to the locations of these lines in the phase diagram for the pure solvent [41]. As a consequence, the solute concentration influences the hole nucleation rate. More importantly, the solute particles may also destabilise the dewetting fronts. As a result, one may find strongly ramified structures in all three systems [23, 25, 40, 42]. A selection of images exhibiting some of the possible structures is displayed in Fig. 1 .

For volatile solvents, the contact lines retract even for wetting fluids. It has been found that such evaporatively receding contact lines may deposit very regular line or ring patterns parallel to the moving contact line [24, 43]. The deposition of a single ring of colloids from a evaporating drop of colloidal suspension is well known as the 'coffee stain effect' [44]. Detailed investigations reveal the emergence of rich structures including multiple irregular rings, networks, regular droplet patterns, sawtooth patterns, Sierpinski carpets, and - in the case of DNA - liquid crystalline structures [22, 30, 45,-49]. The deposition of regularly spaced straight lines orthogonal to the moving contact line has also been reported [50]. Droplet patterns may as well be created employing solvent-induced dewetting of glassy polymer layers below the glass transition temperature [51-53].

Note that the dewetting of pure volatile liquids has also been studied experimentally [54] and theoretically [55-58]. In this case, different contact line instabilities have been observed for evaporating liquid drops [59, 60].

In the present article we review and preview the experiments and in particular the various modelling approaches for dewetting suspensions of (nano-)particles in volatile partially wetting solvents. After reviewing the basic experimental results in Section II] we discuss in Section III several theoretical approaches. In particular, we present a kinetic Monte Carlo model in Section III A. a dynamic density functional theory in Section IIIB, and a thin film evolution equation in Section III C. Finally, we conclude in Section IV by discussing advantages and shortcomings of the individual approaches and future challenges to all of them.

\section{EXPERIMENT WITH NANOPARTICLE SOLUTIONS}

We focus on experiments that use monodisperse colloidal suspensions of thiol-passivated gold nanoparticles in toluene [33, 34, 37,-40,61]. The gold core of $2-3 \mathrm{~nm}$ diameter is coated by a layer of alkyl-thiol molecules. The length of the carbon backbone of the thiol used in the experiments ranges from 6 to 12 carbon atoms $\left(C_{6}\right.$ to $\left.C_{12}\right)$ [40]. By varying the chain length, one can control 

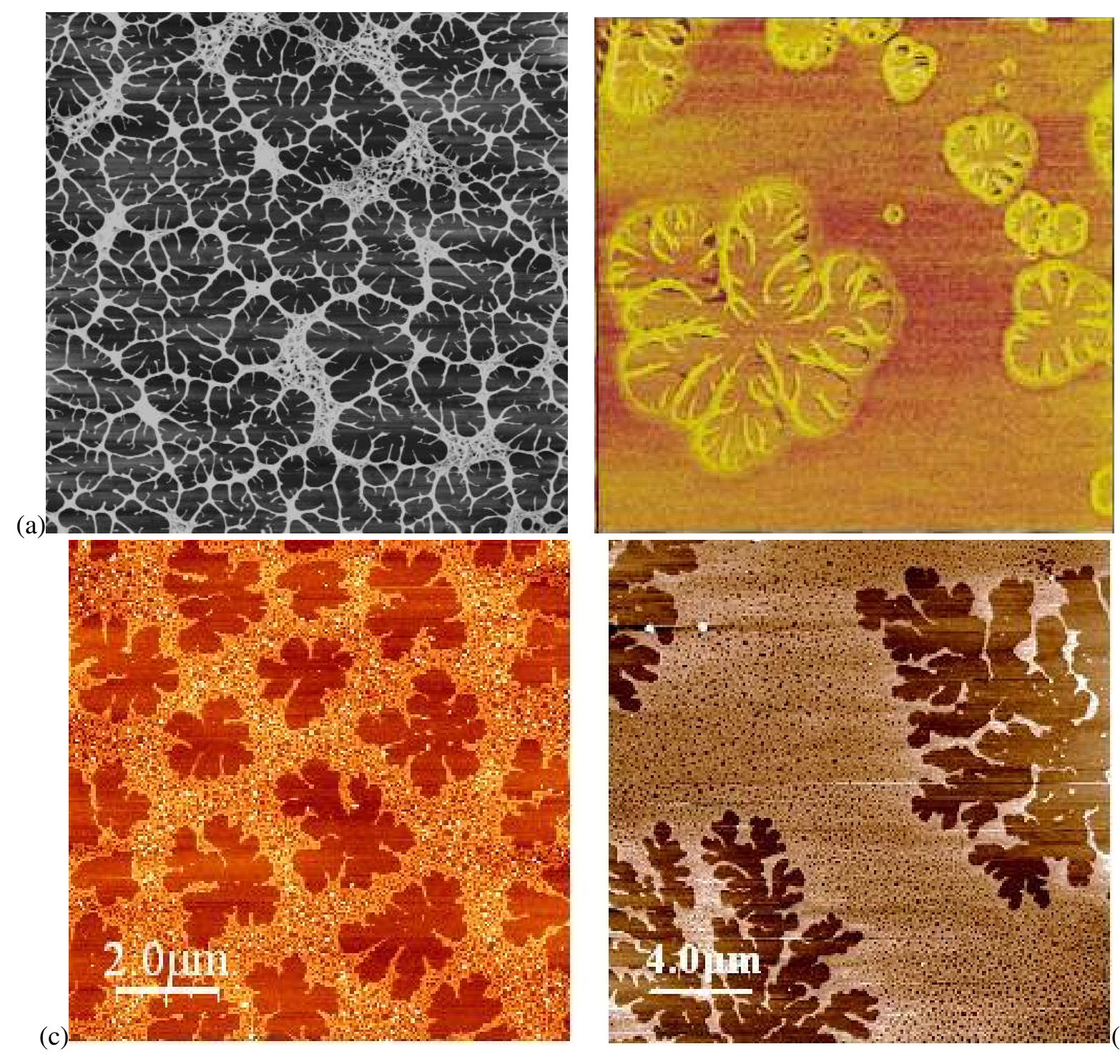

(b)

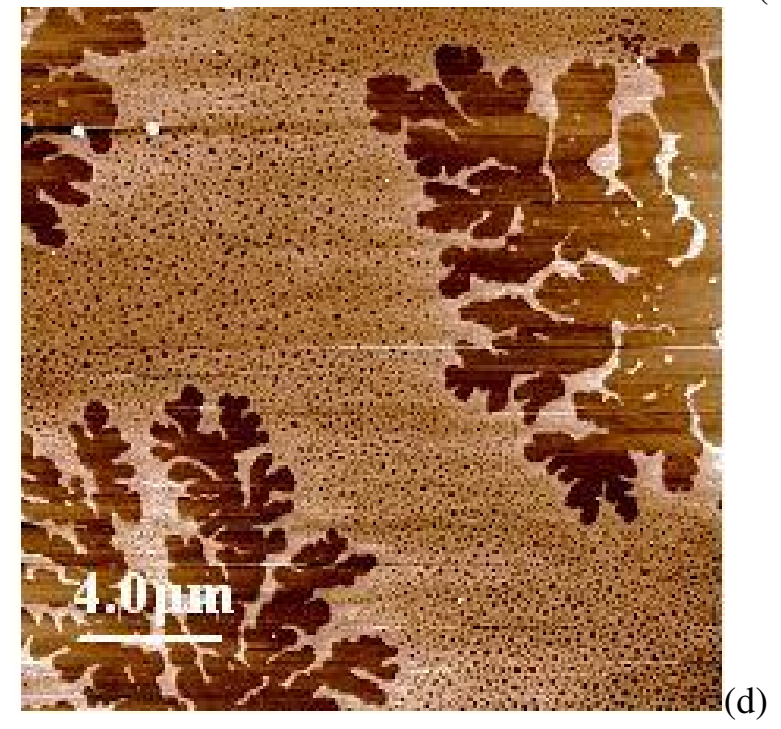

FIG. 1: (Colour online) Images of strongly ramified dewetting structures obtained using Atomic Force Microscopy in the case of (a) an aqueous collagen solution on graphite (courtesy of U. Thiele, M. Mertig and W. Pompe; see also Ref. [42]. Image size: $5 \mu \mathrm{m} \times 5 \mu \mathrm{m}$ ); (b) poly(acrylic acid) in water spin-coated onto a polystyrene substrate (reprinted with permission of John Wiley \& Sons, Inc. from Ref. [23]; copyright John Wiley \& Sons, Inc. 2002; Image size: $2.5 \mu \mathrm{m} \times 2.5 \mu \mathrm{m}$ ); and in both (c) and (d), a solution of gold nanoparticles in toluene, spin-coated onto native oxide terminated silicon substrates (scale bars given in panels). In all the images the lighter areas correspond to the deposited solute and the dark areas to the empty substrate. 
to a certain extent the particle-particle attraction. Normally, the solution is deposited on to a plain silicon substrate that is covered by the native oxide layer only [34]. However, one may locally change the wetting behaviour of the solvent by further oxidising the substrate [38]. By adding excess thiol one can also vary the properties of the solvent [40].

Two different procedures are employed for the deposition of the solution on to the substrate: spincoating or a meniscus technique [61, 62]. The choice is important as it strongly influences the evaporation rate and, as a result, the pattern formation process. When using spin-coating, one finds that directly after deposition, evaporation competes with dewetting until all the solvent has evaporated. The resulting deposits of nanoparticles are imaged by atomic force microscopy (AFM). For spin-coated films, the evaporation rate is high and structuring is normally finished before the spincoater is stopped. Conversely, the solvent evaporation rate is strongly decreased when employing the meniscus technique [61], i.e., by depositing a drop of solution on a Teflon ring that is wetted by the solvent. This allows for a better control of the process and enables the use of contrast-enhanced microscopy to observe the dewetting process in situ [40]. All pattern formation is confined to the region of the receding contact line of toluene, silicon and air. With both techniques one may find mono-modal or bi-modal polygonal networks [34], labyrinthine spinodal structures, or branched patterns (see Fig. 11). The meniscus technique allows for the study of branched structures in a more controlled manner. The work in Ref. [40] indicates that fingering strongly depends on the interaction strength of the particles, i.e., on the chain length of the thiol molecules coating the gold cores. For short chains $\left(\mathrm{C}_{5}\right.$ and $\left.\mathrm{C}_{8}\right)$ no formation of branched structures is observed. At similar concentrations, well-developed branched structures are formed for longer chains $\left(\mathrm{C}_{10}\right.$ and $\left.\mathrm{C}_{12}\right)$. For even longer chains $\left(\mathrm{C}_{14}\right)$, however, one again finds less branching. It also depends on the amount of excess thiol in the solvent (for details see Ref. [40]).

When following the evolution of the branched patterns in situ (see the complementary video material of Ref. [40]), one clearly observes that different processes occur on different lenght scales. First, a macroscopic dewetting front recedes, leaving behind a seemingly dry substrate. The macroscopic front can be transversely unstable resulting in large-scale $(>100 \mu \mathrm{m})$ strongly anisotropic fingered structures. For fronts that move relatively quickly these macroscopic structures cover all the available substrate. However, when at a later stage the macroscopic front becomes slower, those fingers become scarce and 'macroscopic fingering' finally ceases. At this stage it is possible to appreciate that the seemingly dry region left behind by the front is not at all dry, but covered by an ultrathin 'postcursor' film that is itself unstable. The thickness of this film 
is similar to the size of the nanoparticles. At a certain distance from the macroscopic front, the ultrathin film starts to evolve a locally isotropic pattern of holes. The holes themselves grow in an unstable manner resulting in an array of isotropically branched structures as shown, e.g., above in Fig. 1. This indicates that at least some of the patterns described in the literature may have arisen from processes in similar ultrathin 'postcursor' films.

The existence of the ultrathin 'postcursor' film is an experimental finding that can be drawn on when choosing a theoretical approach to account for the pattern formation (see below). Note however, that at the moment there exists no explanation for its existence. A possible hypothesis is that the substrate strongly attracts the nanoparticles. As a result they form a dense suspension layer having a thickness roughly equal to the diameter of the nanoparticles. The observed mesoscopic dewetting front then actually correspond to an autophobic dewetting of a low concentration suspension from the higher concentration suspension on the surface of the substrate.

\section{MODELLING APPROACHES}

Models of dewetting thin films of pure liquids or polymers are often based on thin film hydrodynamics. Starting from the Stokes equations, together with continuity and boundary conditions at the substrate and free surface, one applies a long-wave approximation (assuming small surface slopes and contact angles) [8, 63] and obtains a non-linear evolution equation for the film thickness profile $h(x, y, t)$. In the case of volatile liquids one finds [55-58, 64]

$$
\partial_{t} h=\nabla \cdot\left[Q_{\mathrm{c}} \nabla \frac{\delta F}{\delta h}\right]-Q_{\mathrm{e}} \frac{\delta F}{\delta h},
$$

with the mobility functions $Q_{\mathrm{c}}(h)=h^{3} / 3 \eta \geq 0$ (assuming Poiseuille flow in the film and no slip at the substrate; $\eta$ is the dynamic viscosity) and $Q_{\mathrm{e}} \geq 0$ for the convective and evaporative part of the dynamics, respectively. $Q_{\mathrm{e}}$ is a rate constant that can be obtained from gas kinetic theory or from experiment [57]. Note that Eq. (1) only applies if the pressure in the vapour above the film is close to the saturation pressure. For alternative expressions that are used to describe the non-conserved evaporative dynamics see, e.g., Refs. [56, 57, 65-69]. Finally, $\nabla=\left(\partial_{x}, \partial_{y}\right)$, and $\partial_{t}, \partial_{x}$ and $\partial_{y}$ denote partial derivatives w.r.t. time and the coordinates.

Focusing on the influence of capillarity and wettability only, the energy functional $F[h]$ is given by

$$
F[h]=\int d x \int d y\left[\frac{\gamma}{2}(\nabla h)^{2}+f(h)-\mu h\right]
$$


where $\gamma$ is the liquid-gas surface tension and $f(h)$ is a local free energy term that describes the wettability of the surface. Since $\mu$ corresponds to a chemical potential, the term $\mu h$ may either bias the system towards the liquid or towards the gas state. The variation of $F$ w.r.t. $h$ gives the pressure. It contains the curvature (Laplace) pressure $-\gamma \Delta h$ and the disjoining pressure $\Pi(h)=-\partial_{h} f(h)$. Many different forms for the latter are in use (see, e.g., Refs. [4, 8, 63, 70-73]).

For the present system a thin film description using Eq. (1) is not appropriate because the nanoparticles are not taken into account. However, under certain conditions one can augment equation (1) for the evolution of the film thickness by coupling it to an equation for the evolution of the mean particle concentration. The resulting model is able to describe the behaviour of an evaporating solution on the meso- and macroscale. Such an approach is briefly discussed below in Section IIIC We should expect such a model to describe the mesoscopic dewetting front discussed above. However, the theory is less suited to a description of the dewetting dynamics of the ultrathin postcursor film.

The dewetting of the ultrathin film of highly concentrated suspension may be described by a discrete stochastic model such as, for instance, a kinetic Monte Carlo (KMC) model based solely on evaporation/condensation dynamics of the solvent and diffusion of the solute [35, 39, 41]. The validity of this strong assumption regarding the relevant transport processes can be confirmed from an estimate based on Eq. (1): The pressure $p=\delta F / \delta h$ drives convection and evaporation. The convective mobility is proportional to $h^{3}$, i.e., it is large for thick films but decreases strongly with reduced film thickness. The evaporative mobility, however, is a constant, implying that evaporation will dominate below a certain (cross-over) thickness. For the parameter values of Ref. [57] and a small contact angle $(\approx 0.01)$, the cross-over thickness is in the range of 1-5 nanometers. This estimate justifies the neglect of convective transport in a description of the postcursor film and may explain why one has such good agreement between the experimentally observed patterns and the patterns obtained from a purely two-dimensional (single layer) kinetic Monte Carlo model [35]. We introduce the KMC model below in Section III A.

In several respects, however, the kinetic Monte Carlo model is rather simplistic, limiting its potential applications. For instance, the thermodynamic chemical potential as well as any wetting interaction of the solvent with the substrate are collected in a single parameter - an effective chemical potential. This implies that any influence of a disjoining pressure is 'smeared out' over the whole system and that no distinction between the short- and the long-range parts of the disjoining pressure is possible. It is furthermore based on the assumption that evaporation/condensation is 
the dominant dynamic process, but does not allow one to probe this assumption. In Section IIIB we show how one may develop a dynamical density functional theory (DDFT) that describes the system at a similar level to the KMC. However, the DDFT may also be easily extended to include other effects such as fluid diffusion, that the KMC does not incorporate.

\section{A. Kinetic Monte Carlo model}

The kinetic Monte Carlo model for two-dimensional dewetting nanofluids [33] was first proposed in Ref. [35] and extended to include next-nearest neighbour interactions in [37]. The two key assumptions used are: (i) the relevant processes can be mapped on to a two-dimensional lattice gas model, thereby neglecting continuous changes in the thickness of the evaporating film, and (ii) all relevant dynamics results from diffusing nanoparticles and evaporating/condensing solvent. The model builds on an Ising-type model for the liquid-gas phase transition. The surface is divided up into a regular array of lattice sites whose size is dictated by the nanoparticles. One then considers each lattice site to be occupied either by a nanoparticle, liquid or vapour. This effectively maps the system onto a two-dimensional two-component lattice gas having two fields $n$ and $l$. The resulting three possible states of a cell are: liquid $(l=1, n=0)$, nanoparticle $(l=0, n=1)$, and vapour ( $l=0, n=0$, i.e., cell empty). The energy of an overall configuration is given by the hamiltonian

$$
E=-\frac{\varepsilon_{n n}}{2} \sum_{<i j>} n_{i} n_{j}-\frac{\varepsilon_{n l}}{2} \sum_{<i j>} n_{i} l_{j}-\frac{\varepsilon_{l l}}{2} \sum_{<i j>} l_{i} l_{j}-\mu \sum_{i} l_{i}
$$

where $\sum_{<i j>}$ denotes a sum over nearest neighbour pairs and $\varepsilon_{l l}, \varepsilon_{n n}$ and $\varepsilon_{n l}$ are the liquid-liquid, particle-particle and liquid-particle interaction energies, respectively. Fixing the three interaction strength parameters $\varepsilon_{l l}, \varepsilon_{n n}, \varepsilon_{n l}$ and the effective chemical potential $\mu$ determines the equilibrium state of the system. We choose $\varepsilon_{l l}$ as unit of energy - i.e. we set $\varepsilon_{l l}=1$.

The hamiltonian determines the equilibrium state and the energy landscape of the system. However, as the system 'dries in' during the course of the solvent evaporation, the final nanoparticle configurations do not necessarily represent equilibrium structures. This implies that the system dynamics is of paramount importance. It is determined by the possible Monte Carlo moves, their relative frequencies, and the probabilities for their acceptance. Two types of moves are allowed: (i) evaporation/condensation of liquid and (ii) diffusion of nanoparticles within the liquid. A mobility $M$ corresponds to the ratio of cycles of particle and solvent moves and reflects the physical ratio of 
time scales for evaporation and diffusion. A large mobility $M$ indicates fast diffusion as compared to evaporation. A trial move is accepted with the probability $p_{\text {acc }}=\min [1, \exp (-\Delta E / k T)]$ where $k$ is the Boltzmann constant, $T$ the temperature and $\Delta E$ is the change in energy resulting from the potential move. Note that particles are only allowed to move into wet areas of the substrate, i.e., onto cells with $l=1$. This models zero diffusivity of the particles on a dry substrate. The replaced liquid fills the site left by the nanoparticle.

Without nanoparticles, the behaviour of the model is well known as it reduces to the classical two-dimensional Ising model [74]. For $k T<k T_{c} \approx 0.567$ liquid and vapour coexist when $\mu=$ $\mu_{\text {coex }}=-2$. For $\mu>-2[\mu<-2]$ eventually the liquid [vapour] dominates. A straight liquidgas interface will recede [advance] for $\mu<-2[\mu>-2]$, i.e. one finds evaporative dewetting [wetting] fronts. If one starts, however, with a substrate covered homogeneously by the liquid, for $\mu<-2$ the film will dewet via a nucleation or spinodal-like process. If the nanoparticles are present, they form dried-in structures when all the liquid evaporates. The final structures do not normally change any further - at least on short time scales. However, if the liquid wets the particles (i.e. is attracted to the particles), over long times there might be a coarsening of the structures, facilitated by the adsorbed liquid. The dried-in patterns depend on the particular pathway taken by the evaporative dewetting process. They range from labyrinthine to polygonal network structures or holes in a dense particle layer. Some typical patterns are displayed in Fig. 2, for cases when the average surface coverage of the nanoparticles $\rho_{n}^{a v}=0.2$. Panels (a) and (b) result from a spinodal-like and nucleation and growth process, respectively. At first sight they look very similar to the patterns seen for the pure solvent and one might argue that the particles solely act as passive tracers and preserve the transient volatile dewetting structures of the solvent. This was suggested in Refs. [26-28] for dewetting collagen solutions. However, panels (c) and (d) indicate that the particles may at times play a rather more significant role. When the diffusion of the particles is slow, the evaporative dewetting fronts become transversely unstable and may result in strongly ramified patterns. This instability is caused by the nanoparticles. The lower their mobility, the stronger the fingering effect, i.e., there are more fingers in (c) than in (d) because in the latter the mobility is larger.

The front instability is intriguing as it results in strongly branched structures. As the dewetting front moves, new branches are continuously created and existing branches merge at the moving contact line. However, the mean finger number in the streamwise direction of the resulting ramified pattern is a constant. This behaviour is in contrast to the front instabilities found for dewetting 


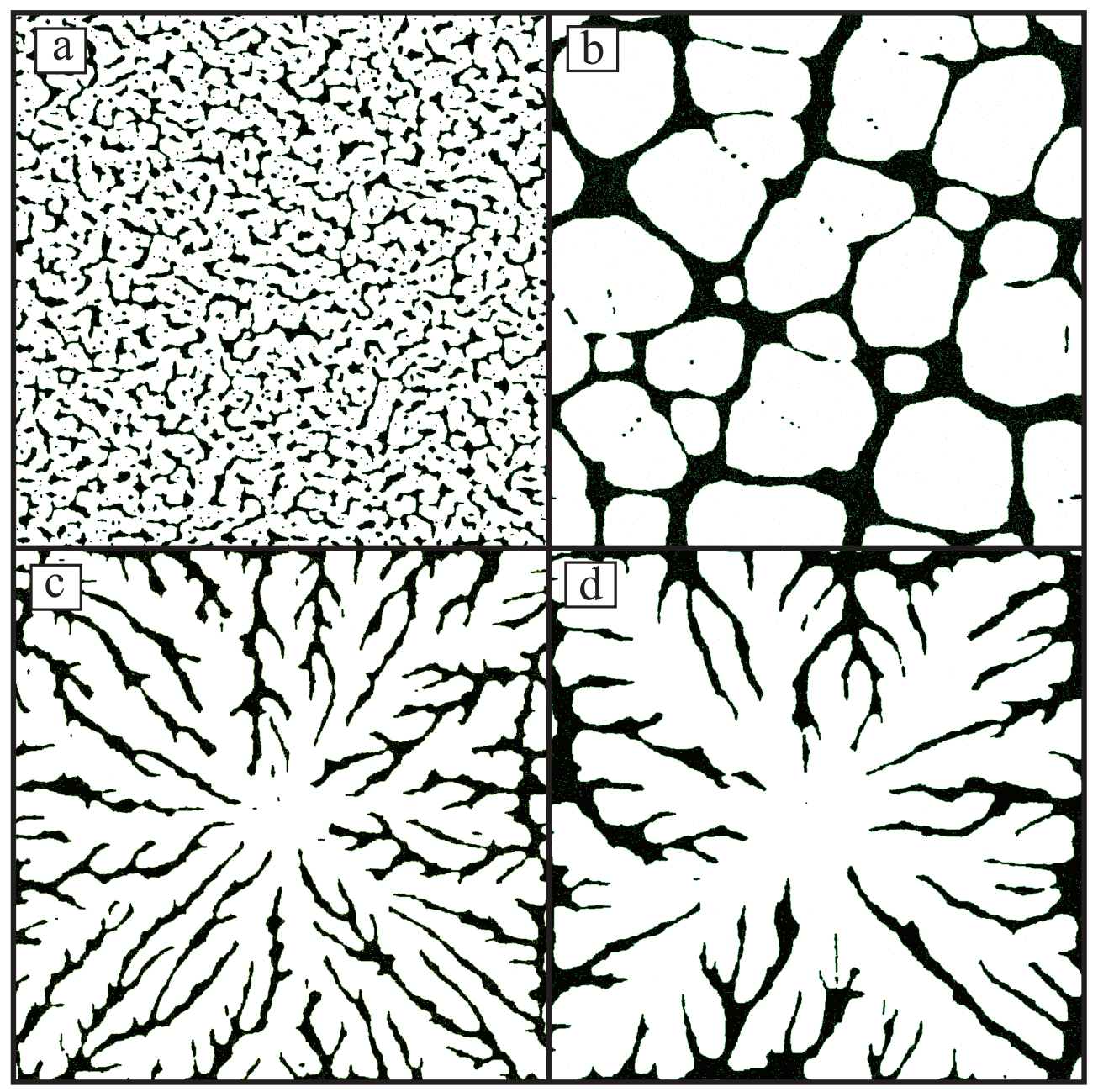

FIG. 2: Typical KMC results for the final dried-in nanoparticle structures resulting from the evaporative dewetting processes of nanoparticle solutions (nanofluids) in the case of (a) a spinodal-like process at $\mu=$ -2.55 , (b) nucleation and growth of holes at $\mu=-2.3$, (c) unstable fronts at $\mu=-2.3$ and low mobility $M=5$, and (d) unstable fronts at $\mu=-2.3$ and medium mobility $M=10$. The starting configuration in (a) and (b) is a homogeneous liquid film with uniformly distributed particles whereas in (c) and (d) a hole at the center is nucleated 'by hand'. The remaining parameters are (a,b) $M=50, \epsilon_{n l}=2.0, \epsilon_{n n}=1.5$, $\rho_{n}^{a v}=0.2, k T=0.3, \mathrm{MC}$ steps $=500$, domain size $1200 \times 1200 ;(\mathrm{c}, \mathrm{d}) \varepsilon_{n n}=2.0, \epsilon_{n l}=1.5, \rho_{n}^{a v}=0.2$, $k T=0.2$, MC steps $=3000$, domain size $1200 \times 1200$. Lattice sites occupied by particles are coloured black, and the empty sites are coloured white. 
polymers which only result in fingers without side-branches [75] or fields of droplets left behind [18].

A quantitative analysis shows that the mean number of fingers depends only very weakly on the average concentration of the nanoparticles $\rho_{n}^{a v}$; only the mean finger width increases with increasing concentration. However, decreasing the mobility (i.e., decreasing the diffusivity of the particles) leads to a much denser finger pattern and also causes the front instability to appear at an earlier stage, i.e., when the front instability is in its initial linear regime, it has a higher growth rate and a smaller characteristic wavelength (cf. Fig. 2(c) and (d)). Decreasing the effective chemical potential (increasing its absolute value) has a similar but less strong effect. For details see [41]. These findings lead to the conclusion that the determining factor for the front instability is the ratio of the time-scales of the different transport processes. In particular, the front becomes more unstable when the velocity of the dewetting front increases as compared to the mean diffusion velocity of the nanoparticles.

If the particle diffusivity is low, the front 'collects' the particles, resulting in a build up of the particles at the front that itself is slowed down. This makes the front unstable and any fluctuation along the front will trigger a transverse instability that results in an evolving fingering pattern. This happens even when the particle-liquid and particle-particle attractive interactions do not favour clustering (i.e. demixing of the liquid and the nanoparticles). In this regime, the instability is a purely dynamic effect and energetics plays no role in determining the number of fingers. We call this the 'transport regime'.

To illustrate the influence of energetics (characterized by the interaction parameters $\varepsilon_{i j}$ ) on fingering in Fig. 3 we display the dependence of the mean finger number on particle-liquid interaction strength $\varepsilon_{n l}$. For $\varepsilon_{n l} \geq 1.5$ the mean finger number $<f>$ is nearly constant; this is the transport regime. However, on decreasing $\varepsilon_{n l}$ below 1.5, we observe a marked increase in the value of $\langle f>$, indicating that energy plays an important role in determining the number of fingers in this regime. In this parameter range, demixing of particles and liquid occurs at the moving front and increases its transverse instability. In this 'demixing regime', the wavelength of the fingering instability is determined by the dynamics and the energetics of the system. Decreasing $\varepsilon_{n l}$ further (below 1.4 in Fig. 3) one first observes in regime (iii) a slight decrease in the average finger number. This is a geometric effect resulting from our one-dimensional finger counting routine: The fingers increasingly break up and the dried-in pattern looks progressively isotropic. In regime (iv), the measure $\langle f\rangle$ does not represent a finger number but instead indicates a decrease in the typical 
distance between particle clusters resulting from the demixing process that occurs already in the bulk liquid and is not related to the front instability at all. Note that one finds a similar sequence of regimes (i) to (iv) when increasing the particle-particle interaction strengths for fixed $\varepsilon_{n l}$ (see Ref. [41]) for further details.

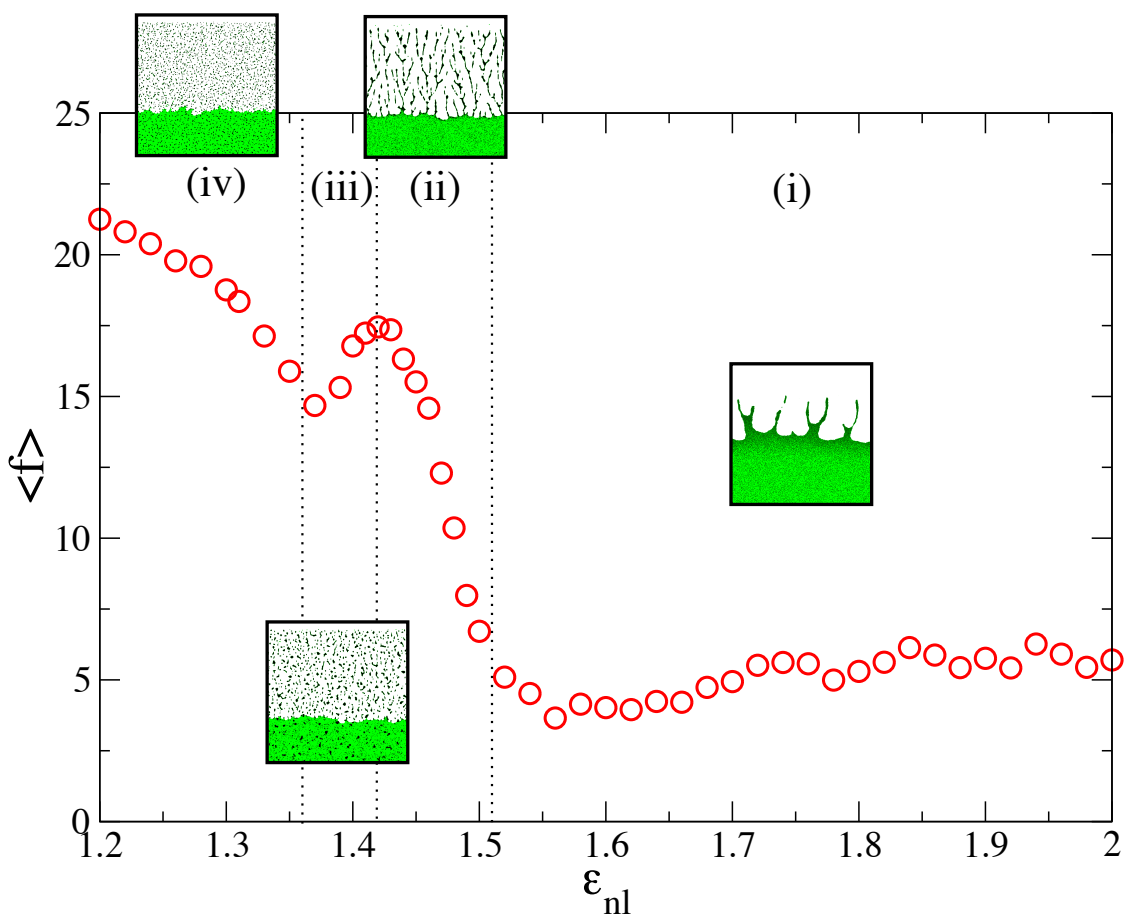

FIG. 3: (Colour online) Dependence of the mean finger number left behind by the unstable dewetting front on the particle-liquid interaction strength $\varepsilon_{n l}$. The regions marked (i) to (iv) are discussed in the main text. The insets display typical snapshots obtained in the four different regions. Particles are black, liquid is grey (green online) and the empty substrate is white. The remaining parameters are $k T=0.2$, $M=20, \mu=-2.2, \rho_{n}^{a v}=0.1, \epsilon_{n n}=2.0$, domain size $1200 \times 1200$. For the insets, from left to right, $\epsilon_{n l}=1.2,1.4,1.45,1.8$.

We note also that the fingering process may be viewed as self-optimising the front motion - i.e. the front keeps its average velocity constant by expelling particles into the fingers. A similar effect exists for dewetting polymer films [18], where liquid is expelled from the growing moving rim which collects the dewetted polymer. There, the surplus liquid is left on the surface as a droplet pattern.

The kinetic Monte Carlo model is a very useful tool that helps one to understand the pattern formation in drying nanoparticle suspensions. One has, however, to keep in mind the restrictions 
on the model (see above). The purely two-dimensional character of the KMC was extended to a 'pseudo three-dimensional' one by making the effective chemical potential dependent on the mean liquid coverage [38]. As the latter is related to a mean film thickness, this corresponds to the introduction of a 'global' thickness-dependent disjoining pressure into the evaporation term without an explicit consideration of a film thickness. The amended model can reproduce bimodal structures that are beyond the scope of the purely two-dimensional model [38, 39]. Fully threedimensional models are also discussed in the literature [76, 77].

\section{B. Dynamical Density Functional theory}

The limitations of the kinetic Monte Carlo model introduced in the previous Section are related to its character as a two-dimensional lattice gas with only three states: gas, liquid or particle. This implies that (i) no liquid can be transported to a site on the surface already filled with liquid, i.e., diffusion of the liquid can not be incorporated in a sensible way and (ii) one is not able to distinguish between the influence of the short- and the long-range parts of the interactions with the substrate, as all such interactions are absorbed into the effective chemical potential.

However, using dynamical density functional theory (DDFT) [78-83] one can develop a model for the processes in the ultrathin postcursor film without these limitations, although here we limit ourselves to developing the theory at the level of the KMC and solely discuss how to extend it to incorporate the influence of the liquid diffusion over the surface. Such a DDFT model describes the coupled dynamics of the density fields of the liquid $\rho_{l}$ and the nanoparticles $\rho_{n}$. The densities $\rho_{l}$ and $\rho_{n}$ are defined as the probabilities of finding a given lattice site on the surface to be occupied by a film of liquid or by a nanoparticle, respectively. Note that the probability densities correspond to number densities as we use the lattice spacing $\sigma=1$ as our unit of length.

To develop the DDFT, one must first derive the underlying free energy functional $F\left[\rho_{l}, \rho_{n}\right]$, and secondly, devise dynamical equations for both density fields that account for the conserved and the non-conserved aspects of their dynamics, i.e., transport and phase change processes, respectively. For a system governed by the hamiltonian (3), we may construct a mean-field (Bragg-Williams) approximation for the free energy of the system [78, 84] which contains an entropic contribution and contributions from the interactions between the different species (nanoparticles and liquid). The free energy is a semi-grand free energy, since the liquid is treated grand canonically (it is coupled to a reservoir with chemical potential $\mu$ ), whereas the nanoparticles are treated in the 
canonical ensemble. The free energy functional is first defined on the original KMC lattice. However, after re-writing the interaction terms employing gradient operators [78] one finally obtains the free energy functional for a continuous system

$$
F\left[\rho_{l}, \rho_{n}\right]=\int \mathrm{d} \mathbf{r}\left[f\left(\rho_{l}, \rho_{n}\right)+\frac{\varepsilon_{l l}}{2}\left(\nabla \rho_{l}\right)^{2}+\frac{\varepsilon_{n n}}{2}\left(\nabla \rho_{n}\right)^{2}+\varepsilon_{n l}\left(\nabla \rho_{n}\right) \cdot\left(\nabla \rho_{l}\right)-\mu \rho_{l}\right],
$$

where

$$
\begin{aligned}
f\left(\rho_{l}, \rho_{n}\right) & =k T\left[\rho_{l} \ln \rho_{l}+\left(1-\rho_{l}\right) \ln \left(1-\rho_{l}\right)\right] \\
& +k T\left[\rho_{n} \ln \rho_{n}+\left(1-\rho_{n}\right) \ln \left(1-\rho_{n}\right)\right] \\
& -2 \varepsilon_{l l} \rho_{l}^{2}-2 \varepsilon_{n n} \rho_{n}^{2}-4 \varepsilon_{n l} \rho_{n} \rho_{l} .
\end{aligned}
$$

Since the liquid may evaporate from the surface into the vapour above the surface, $\mu$ is the (true) chemical potential of this reservoir and determines the rate of evaporation [condensation] from [to] the surface. Note that normally a free energy of the form in Eq. (4) is obtained by making a gradient expansion of the free energy functional of a continuous system [84]. However, here we have made the mapping from the free energy of the lattice KMC system.

The chemical potential for the nanoparticles may be determined from the functional derivative $\mu_{n}=\delta F\left[\rho_{n}, \rho_{l}\right] / \delta \rho_{n}(\mathbf{r})$. In equilibrium it is constant throughout the system, but it may vary spatially in a non-equilibrium system, i.e., $\mu_{n}=\mu_{n}(\mathbf{r}, t)$. We assume that the dynamics of the nanoparticles is governed by the thermodynamic force $\nabla \mu_{n}$-i.e. that the nanoparticle current is $\mathbf{j}=-M_{n} \rho_{n} \nabla \mu_{n}$, where $M_{n}\left(\rho_{l}\right)$ is a mobility coefficient that depends on the local density of the liquid. Combining this expression for the current with the continuity equation, we obtain the following evolution equation for the nanoparticle density profile

$$
\frac{\partial \rho_{n}}{\partial t}=\nabla \cdot\left[M_{n} \rho_{n} \nabla \frac{\delta F\left[\rho_{n}, \rho_{l}\right]}{\delta \rho_{n}}\right] .
$$

Note that this equation of motion may also be obtained by assuming that the nanoparticles have over-damped stochastic equations of motion [80-83]. Here, we assume that $M_{n}\left(\rho_{l}\right)=\alpha \Theta_{s}\left(\rho_{l}-\right.$ $0.5)$, where $\Theta_{s}(x)$ is a continuous function that switches smoothly from the value 0 to the value 1 at $x=0$ (i.e. it is essentially a smooth analogue of the Heaviside function). This ensures that the nanoparticles are immobile when the local liquid density is small (dry substrate) and have a mobility coefficient $\alpha$ when $\rho_{l}$ is high (wet substrate).

For the evolution of the liquid density distribution we assume that the liquid is able to evaporate from the surface into the vapour (reservoir) above the surface (non-conserved dynamics) and may 


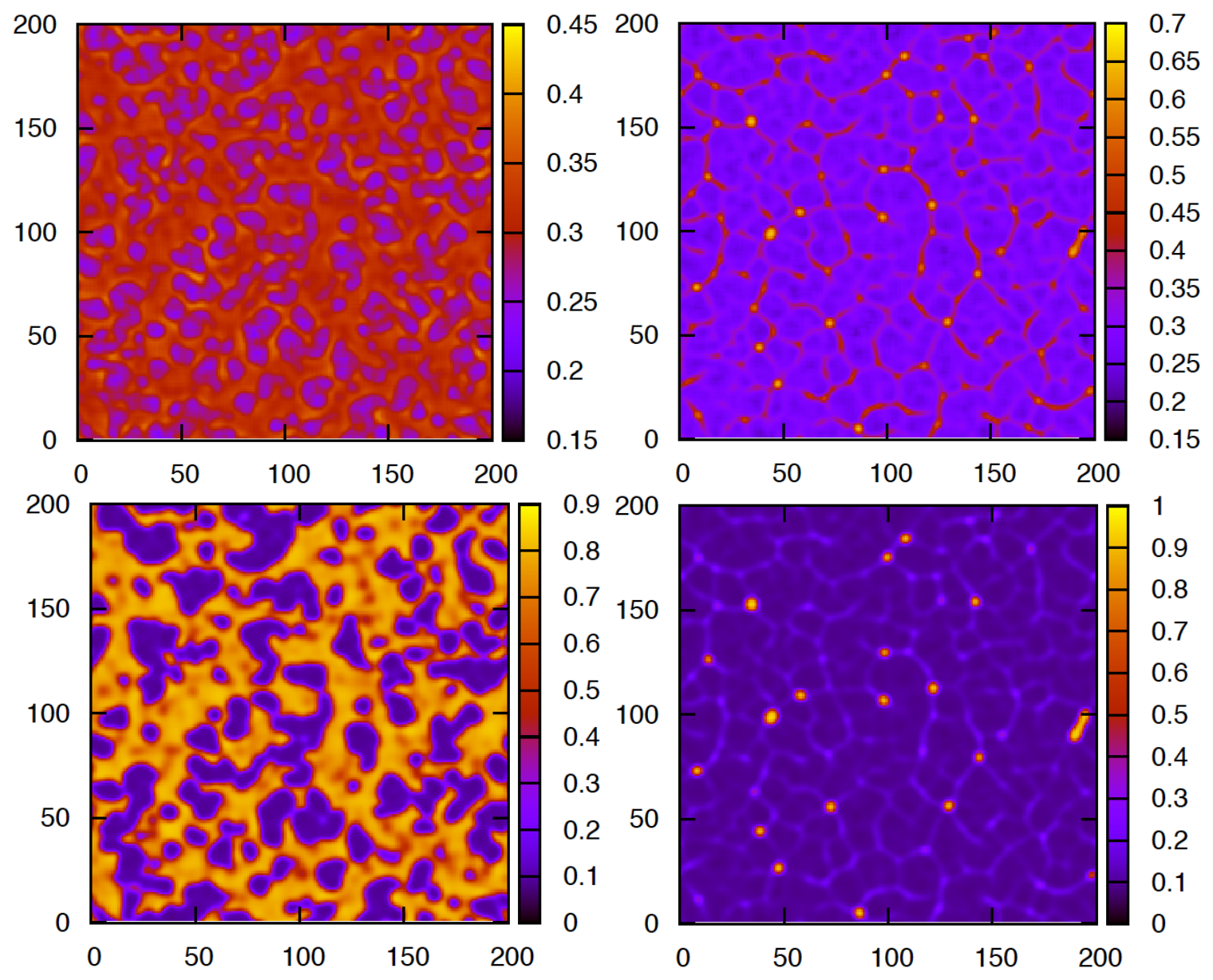

FIG. 4: (Colour online) Density profiles for the situation where the substrate is covered by nanoparticles with average density $\rho_{n}^{a v}=0.3$. The top row are the nanoparticle density profiles and the bottom row are the corresponding liquid density profiles at the times $t / t_{l}=8$ (left) and 80 (right), where $t_{l}=1 / k T M_{l}^{\mathrm{nc}} \sigma^{2}$. The parameters are $k T / \varepsilon_{l l}=0.8, \varepsilon_{n l} / \varepsilon_{l l}=0.6, \varepsilon_{n n}=0, \alpha=0.4 M_{l}^{\mathrm{nc}} \sigma^{4}, M_{l}^{\mathrm{c}}=0, \rho_{l}(t=0)=0.9 \pm \xi$ (where $\xi$ represents white noise of amplitude 0.05$)$ and $\left(\mu-\mu_{\text {coex }}\right) / k T=-0.88$, where the liquid exhibits spinodal decomposition-evaporation.

also diffuse over the substrate (conserved dynamics). The conserved part is treated along the lines developed above for the nanoparticles. For the non-conserved part we assume a standard form [85], i.e., the change in time of $\rho_{l}$ is proportional to $-\left(\mu_{\text {surf }}(\mathbf{r}, t)-\mu\right)=-\delta F\left[\rho_{n}, \rho_{l}\right] / \delta \rho_{l}(\mathbf{r})$ where $\mu_{\text {surf }}(\mathbf{r}, t)$ is the local chemical potential of the liquid at the point $\mathbf{r}$ on the surface at time $t$. This gives the evolution equation for the liquid density

$$
\frac{\partial \rho_{l}}{\partial t}=\nabla \cdot\left[M_{l}^{\mathrm{c}} \rho_{l} \nabla \frac{\delta F\left[\rho_{n}, \rho_{l}\right]}{\delta \rho_{l}}\right]-M_{l}^{\mathrm{nc}} \frac{\delta F\left[\rho_{n}, \rho_{l}\right]}{\delta \rho_{l}},
$$

where we assume that the coefficients $M_{l}^{\mathrm{c}}$ and $M_{l}^{\mathrm{nc}}$ are constants. 


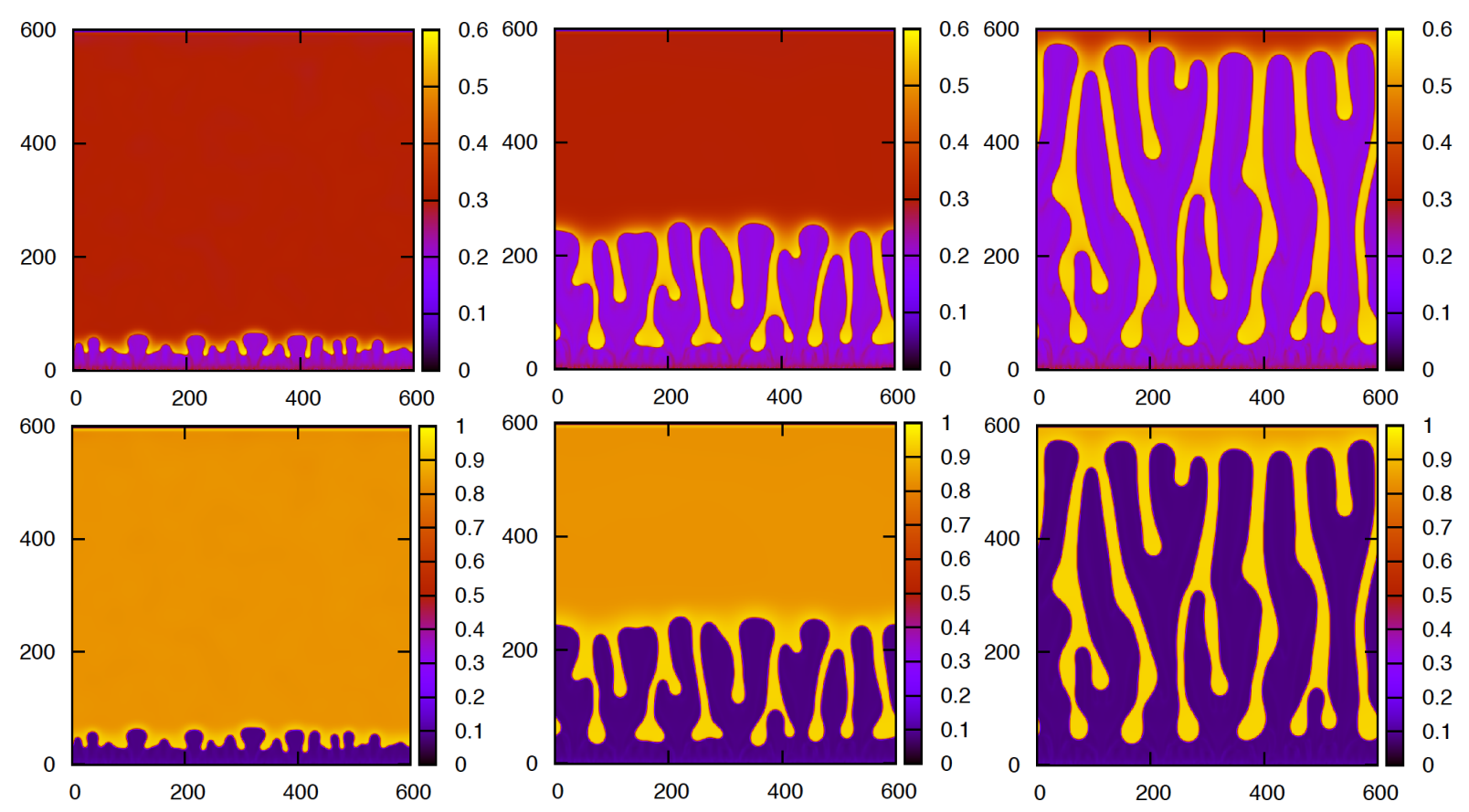

FIG. 5: (Colour online) Density profiles for the situation where the substrate is covered by nanoparticles with average density $\rho_{n}^{a v}=0.3$ and with the liquid excluded from the region $y<0$. The top row shows the nanoparticle density profiles and bottom row the corresponding liquid density profiles at the times $t / t_{l}=1000$ (left), 10000 (middle) and 30000 (right), where $t_{l}=1 / k T M_{l}^{\text {nc }} \sigma^{2}$. The parameters are $k T / \varepsilon_{l l}=0.8, \varepsilon_{n l} / \varepsilon_{l l}=0.6, \varepsilon_{n n}=0, \alpha=0.2 M_{l}^{\text {nc }} \sigma^{4}, M_{l}^{\mathrm{c}}=0, \rho_{l}(t=0)=0.9 \pm \xi$ (where $\xi$ represents white noise of amplitude 0.05$)$ and $\left(\mu-\mu_{\text {coex }}\right) / k T=-0.78$.

This theory allows us to study the time evolution of the evaporating film of nanoparticle suspension without some of the restrictions of the kinetic Monte Carlo model. Here, however, we illustrate its application in similar parameter regimes as used above for the KMC. We focus on two examples: (i) the spinodal dewetting of a initially flat film of nanoparticle suspension characterised by constant $\rho_{l}$ and $\rho_{n}$ (Fig. 4); and (ii) the retraction of a dewetting front that is unstable with respect to a fingering instability (Fig. 5).

Fig. 4 presents two pairs of snapshots from a purely evaporative dewetting process deep inside the parameter region of the phase diagram where spinodal dewetting occurs. For small times the film becomes unstable showing a typical spinodal labyrinthine pattern with a typical wavelength. The nanoparticles concentrate where the remaining liquid is situated. However, they are 'slow' in their reaction: when $\rho_{l}$ already takes values in the range $0.08-0.83$, the nanoparticle concentration has only deviated by about $25 \%$ from its initial value. The film thins strongly forming many 
small holes. The competition for space results in a fine-meshed polygonal network of nanoparticle deposits. The concentration of particles is much higher at the network nodes - an effect that can not been seen within the KMC model. As the particles attract the liquid there remains some liquid on the substrate where the nanoparticles are.

Fig. 5 gives snapshots of the evolution of a fingering instability for a retracting dewetting front. At early times the straight front shows a rather short-wave instability, about 16 wiggles can be seen. However, they are only a transient: the finger pattern coarsens rapidly till only about 7 fingers remain. The fingering then becomes stationary, i.e., just as in the KMC, the mean finger number remains constant, although new branches are continuously created and old branches join each other. In general, the results on fingering agree well with results obtained using the KMC model [41]. From this we conclude that jamming of discrete particles is not a necessary factor for causing the instability, since the fingering is seen here in a continuum model with a diffusion constant that is independent of the nanoparticle concentration. The DDFT is better suited than the KMC for investigations of the early instability stages: they are more easy to discern without the discrete background noise of the KMC. Furthermore, one may perform a linear stability analysis of the one-dimensional undisturbed streamwise front profiles with respect to transverse perturbations (in analogy to the approach used in Refs. [19, 86, 87]).

\section{Thin film hydrodynamics}

The previous two sections focused on two approaches to describe the experimentally observed patterning dynamics in the ultrathin postcursor film left behind by a mesoscopic receding dewetting front. Although both the kinetic Monte Carlo model and the dynamical density functional theory are able to describe well the processes in the ultrathin film, they can not be employed to describe mesoscale hydrodynamics. A relatively simple model for the latter can be derived in the framework of a long-wave or lubrication equation [8, 63]. We will illustrate here the approach by considering an isothermal situation where the nanoparticles are not surface active, i.e., they do not act as surfactants. For a model incorporating the effects of latent heat generation and surface-

active particles resulting in thermal and solutal Marangoni stresses, see Ref. [88]. A description of spreading particle solutions incorporating a structural disjoining pressure has also been considered [89]. For related work on particle-laden film flow on an incline see Refs. [90, 91].

One starts from the Stokes equations, together with continuity, no-slip boundary conditions at the 
substrate and force equilibria at the free surface, and applies a long-wave approximation. Under the assumption that concentrations equilibrate rapidly over the film thickness, we obtain coupled non-linear evolution equations for the film thickness profile $h(x, t)$ and the amount of nanoparticles per unit length $h_{p}=\phi h$, where $\phi$ is the volume concentration of the nanoparticles. Note, that $h_{p}$ corresponds to the local thickness of the nanoparticle layer when all the solvent is evaporated. The resulting evolution equation for the film thickness is Eq. (1) above and focusing on the influence of particle-independent capillarity and wettability only, the energy functional $F[h]$ is given by Eq. (2) above. Note that the viscosity $\eta$ depends on the particle concentration. Following Refs. [88, 89, 91, 92] we use the Quemada law for dense suspensions [93-95]

$$
\eta(\phi)=\eta_{0}\left(1-\frac{\phi}{\phi_{c}}\right)^{-2}
$$

where $\phi_{c}=0.64$ corresponds to random close packing of spherical particles. For the nanoparticle volume per length $h_{p}=\phi h$ one obtains the following evolution equation:

$$
\partial_{t}(\phi h)=\nabla \cdot\left[\phi Q_{\mathrm{c}} \nabla \frac{\delta F}{\delta h}\right]+\nabla \cdot[D(\phi) h \nabla \phi],
$$

where the particle concentration dependent diffusion coefficient $D(\phi)$ is related to the viscosity by the Einstein relation $D(\phi)=k T / 6 \pi R \eta(\phi)$, where $R$ is the radius of the nanoparticles [96].

We illustrate results obtained employing this thin film theory using the single example of a receding dewetting front for a partially wetting film. We use the disjoining pressure and material constants for the liquid considered in Ref. [57], where the evaporative and convective dewetting of a film of volatile liquid is studied. We add, however, the nanoparticles to the system. The expression that we employ for the local free energy term in Eq. (2) is:

$$
f(h)=\frac{S_{L W} d_{0}^{2}}{h^{2}}+S_{P} \exp \left(\frac{d_{0}-h}{l_{0}}\right),
$$

where the parameters characterising the interaction between the liquid film and the surface are the apolar and polar spreading coefficients $S_{L W}$ and $S_{P}$, respectively, the Debye length $l_{0}$ and the Born repulsion length $d_{0}$ [57]. The resulting disjoining pressure $\Pi=-\partial_{h} f(h)$ allows for a stable precursor film (thickness $h_{\text {precursor }}$ ) and also has a second (larger) thickness $\left(h_{0}\right)$ that corresponds to a secondary minimum of the underlying energy functional. See Refs. [11, 97] for studies of film and drop states for similar disjoining pressures. Our results are calculated for a system where the profiles only vary in one Cartesian direction $(x)$, corresponding to a straight dewetting front. However, our results may also be interpreted as applying to a circular flat drop whose front remains 

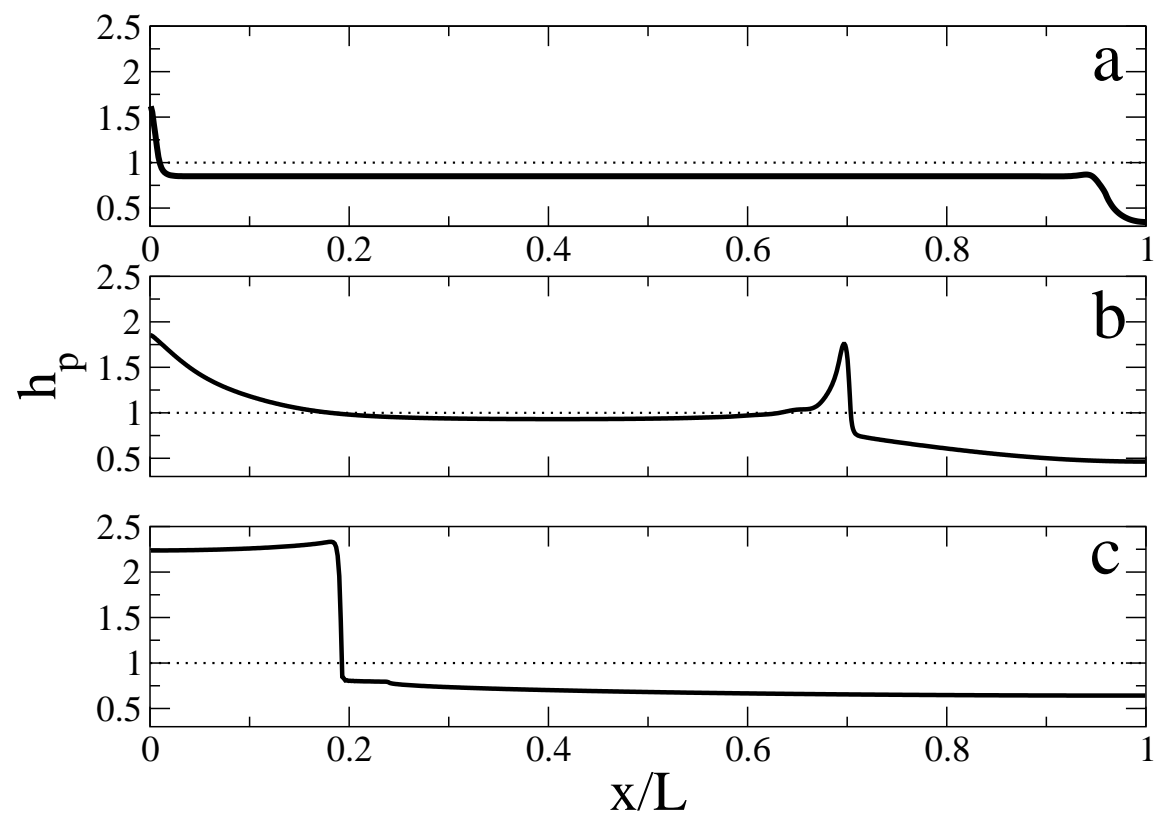

FIG. 6: Profiles of the final dried-in nanoparticle layer for the dewetting of a suspension of nanoparticles in a volatile solvent that partially wets the substrate for (a) high $\left(\Omega=10^{-3}\right)$, (b) medium $\left(\Omega=2 \times 10^{-6}\right)$ and (c) low $\left(\Omega=0.78 \times 10^{-8}\right)$ evaporation rates, for the case when $\chi=H / l_{0}=1.09$, the lateral length scale is $\ell=\sqrt{\gamma / \kappa} H$ with $\kappa=\left(S_{p} / l_{0}\right) \exp \left(d_{0} / l_{0}\right) H$ being an energy scale related to wettability and the vertical length scale is $H=\sqrt{2 S_{L W} / \kappa} d_{0}$. The remaining dimensionless parameters are the evaporation number $\Omega=Q_{e} \eta_{0} \ell^{2} / H^{3}$, the diffusion number $\Gamma=D(0) \eta_{0} / H \kappa=10^{-4}$ and the dimensionless chemical potential $M=H \mu / \kappa=-0.0035$. The system size is $L=19500 \ell$. Film thickness and $h_{p}$ in the plots are scaled by the precursor film thickness.

circular throughout the dewetting and evaporation process. In this case one should interprete the coordinate $x$ as the distance from the centre of the circular film.

We start with a film of height $h_{0}$ of finite length sitting on a precursor film and assume that the film contains nanoparticles at constant concentration $\phi_{0}$. The chosen parameter values ensure that the film of thickness $h_{0}$ is linearly stable. As we do not incorporate noise, no nucleation of additional holes can occur (even with noise the probability would be extremely low). Without evaporation the film dewets 'classically' by a retraction of the initially step-like front. After a short time, surface tension smoothes the profile of the receding front and a capillary rim forms that collects all the 
dewetted liquid. The front recedes until all liquid is collected in a central drop. Since no liquid evaporates $\left[Q_{\mathrm{nc}}=0\right.$ in Eq. (1)], the particle concentration does not change during the process. The situation changes when allowing for evaporation $\left(Q_{\mathrm{nc}}>0\right)$. Now the front may retract by convection and/or evaporation. Evaporation leads to the possibility of a strong increase in the particle concentration at the contact line as evaporation is strongest there. Due to the strong nonlinear dependence of the viscosity on the particle concentration, this may lead to a dramatic decrease of the convective contribution to the front velocity. For moderate evaporation rates, this may result in a (temporary) self-pinning of the front. Within the present basic model, the process can (after complete dry-in) result in three different basic deposition patterns: (i) for very fast evaporation rates, all other processes occur over time scales that are much larger. In particular, the effects of convective redistribution of the liquid are neglectable. As a result one finds that a nearly homogeneous film of nanoparticles of thickness $h_{p}=\phi_{0} h_{0}$ is deposited (see Fig.6(a)). Convection only results in the small heap of material visible at the left hand side of Fig. 6(a). The decrease in $h_{p}$ on the right side of Fig. 6(a) arises due to the diffusion of particles to the right of the initial front position; (ii) for very low evaporation rates, the film dynamics is dominated by convective dewetting as this process acts on a much shorter time scale than evaporation. As a result, all the liquid is collected into a drop before evaporation slowly removes the remaining solvent. Under these conditions most of the nanoparticles are deposited in a single heap (see Fig.6(c)). Depending on the diffusivity, the heap might be highest at the centre or show a depression there; (iii) at intermediate evaporation rates, one may observe the deposition of a nanoparticle ring around a region with a nanoparticle film of much lower height. At the centre deposition might increase again (see Fig. 6(b)).

The most intriguing feature is the ring formation that has been observed experimentally for suspensions of very different particle sizes ranging from nanometers [32, 36, 46, 47] to hundreds of micrometers. Pinning of the contact line and thermal Marangoni effects are often mentioned as necessary conditions for the ring formation. The contact line pinning is often assumed to result from substrate heterogeneities. Film height and concentration profiles at various instants during the dewetting process are displayed in Fig. 7. The profiles are from before, at and after self-pinning of the contact line. In Fig. 8 we display a space-time plot for the complete process. At first, the front recedes in the same manner as when there is no evaporation, but now driven by convection and evaporation. A small capillary rim forms that collects all the dewetted liquid that does not evaporate. The particle concentration slowly increases at the contact line (Fig. 7) (a) and regime 


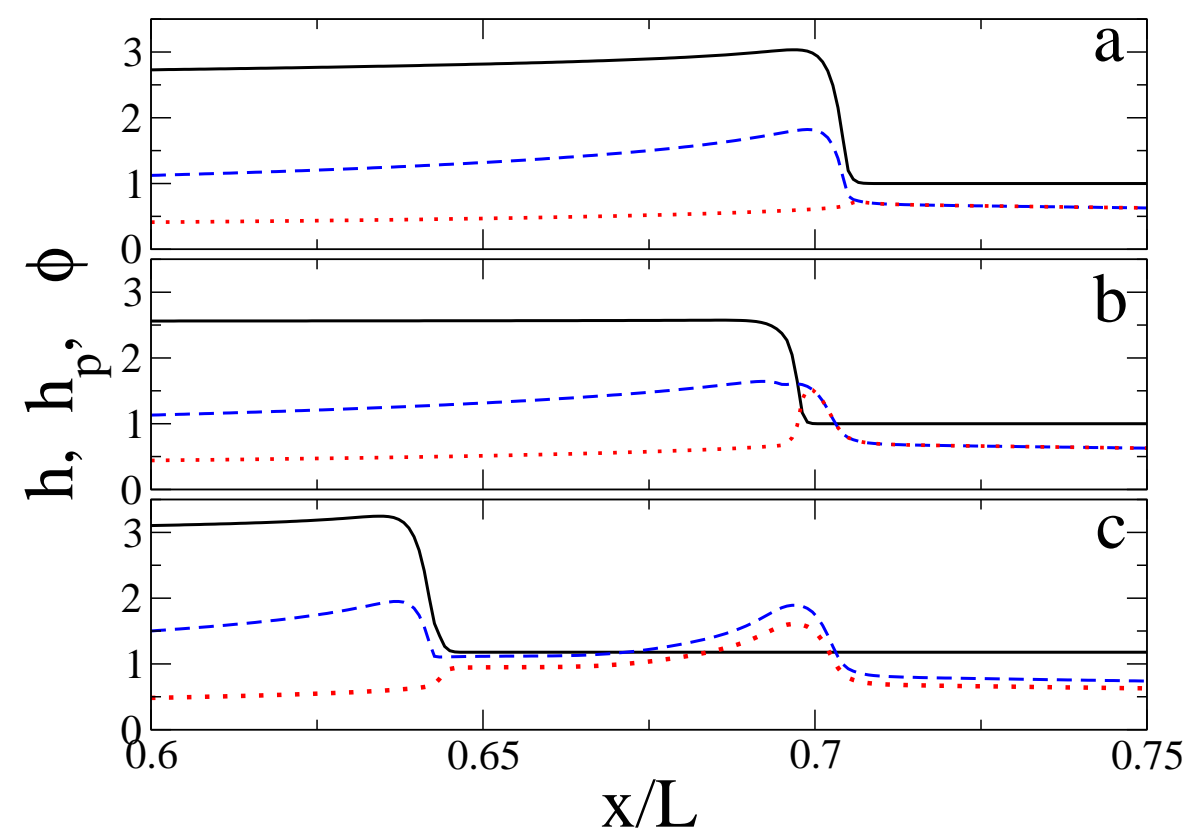

FIG. 7: (Colour online) A sequence of profiles during a dewetting process with competing evaporation and convection that leads to the dried-in ring structure of nanoparticles displayed in Fig. 6(b). Profiles are at (a) before pinning $(t=0.08 T)$, (b) at self-pinning $(t=0.13 T)$, and (c) after depinning $(t=0.29 T)$, where $T=3 \times 10^{10} \tau$ with $\tau=\eta_{0} \gamma H / \kappa^{2}$ ( $T$ is of order of $1 \mathrm{~s}$ ). The film thickness profiles $h$ are the bold solid lines, the nanoparticle concentrations $\phi$ are the dotted lines and the nanoparticle layer height $h_{p}=h \phi$ are the dashed lines. The remaining parameters and scalings are as in Fig. 6(b).

(i) in Fig. 8). The concentration increases further and when it approaches random close packing $\phi_{c}$, the viscosity diverges and the front pins itself. When pinned, further retraction only occurs through evaporation (Fig. 7(b) and regime (ii) in Fig. 8). The front eventually depins and starts to move again, leaving a nanoparticle ring behind (Fig. 7(c) and regime (iii) in Fig. 8). However, the velocity is not as large as at the beginning, owing to the fact that the mean concentration of particles has increased. The remaining particles are transported to the centre and are deposited there when the remaining solvent evaporates (regime (iv) in Fig. 8).

The simple model used here shows, (i) that the contact line stops due to self-pinning by the deposited particles and (ii) the Marangoni effect is not necessary for the ring formation. The model can easily be refined to account for solutal and/or thermal Marangoni effects [88] but self-pinning 

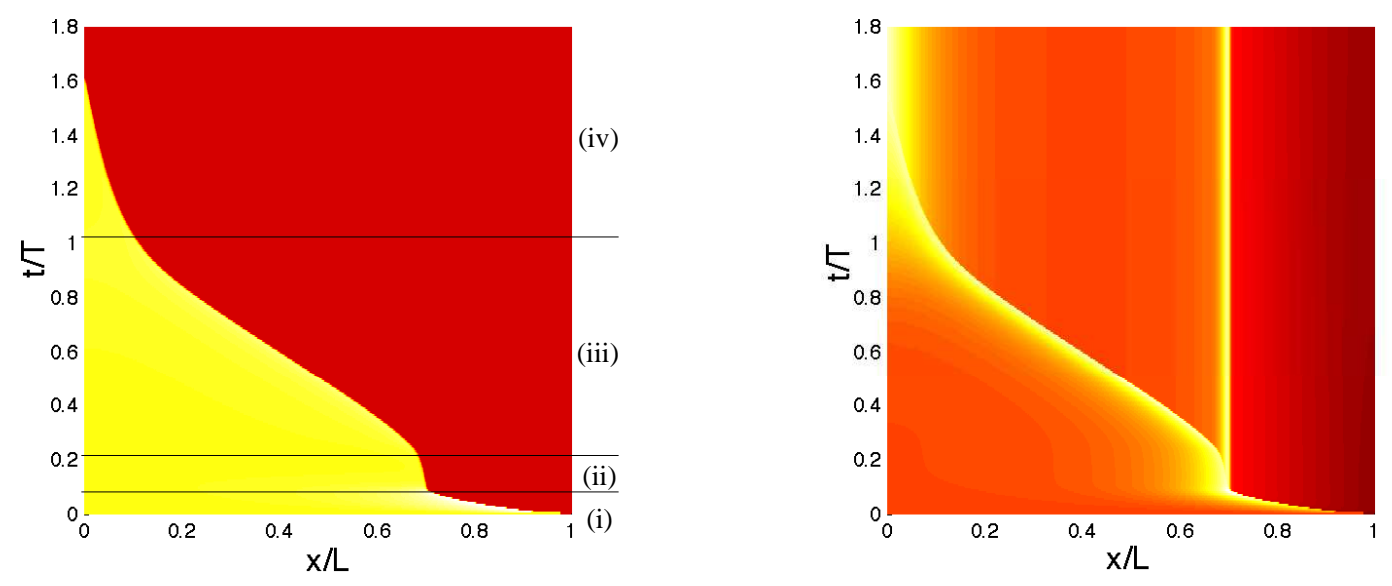

FIG. 8: (Colour online) Space-time plots are given for (left) the film thickness $h$ and (right) the nanoparticle layer height $h_{p}=h \phi$. The plot corresponds to the complete evolution resulting in the ring profile of Fig. 6(b). In both panels bright [dark] parts denote high [low] regions. The prominent central dark-bright border in the left panel indicates the change of the position of the contact line in time. Over time, four regimes can be distinguished: (i) fast motion before pinning, (ii) nearly no front motion during self-pinning, (iii) slow motion after depinning, and (iv) final evaporation from the center.

should also be investigated further in the simple case presented here.

\section{CONCLUSION}

We have discussed recent work on pattern formation processes in films and drops of evaporating suspensions/solutions of polymers and particles. After reviewing experiments on suspensions of thiol-coated gold nanoparticles in toluene we have focused on the modelling of the transport and phase change processes involved. A theoretical approach to the modelling of the hydrodynamics on the mesoscale has been described as well as more microscopic models for the dynamics in the observed nanoscopic 'postcursor' film. In particular, we have introduced (i) a microscopic kinetic Monte Carlo model, (ii) a dynamical density functional theory and (iii) a hydrodynamic thin film model.

The kinetic Monte Carlo model and the dynamical density functional theory can both be used to investigate and understand the formation of polygonal networks, spinodal and branched structures resulting from the dewetting of an ultrathin 'postcursor' film that remains behind the mesoscopic dewetting front. They are, however, not capable of describing the dynamical processes in a meso- 
scopic film. We have seen that the KMC model is able to describe the interplay of solute diffusion within the solvent and solvent evaporation/condensation. It also takes the liquid-liquid, liquidparticle and particle-particle interactions into account and therefore allows us to distinguish different regimes of the transverse (fingering) instability of the evaporative dewetting front: a transport regime where the instability is almost completely independent of the interaction strengths and a demixing regime where particles and liquid demix at the receding front thereby increasing its transverse instability.

The dynamical density functional theory describes the coupled dynamics of the density fields of the liquid and the nanoparticles. In the form described above (i.e. based on the two-dimensional hamiltonian (3) ) we obtain a simple theory that allows us to study the time evolution of the evaporating ultrathin film and also to investigate the influence of processes such as surface diffusion by the liquid, which are not incorporated in the KMC model. However, it is straightforward to extend the theory to consider a fully three-dimensional fluid film, in which one can distinguish between short- and long-range interactions of solvent and/or solute with the substrate. We have, however, restricted the examples given here to situations that can also be described using the KMC model. A further exploration will be presented elsewhere.

Finally, we have discussed a simple thin film model for the hydrodynamics on the mesoscale. It results from a long-wave approximation and consists of coupled evolution equations for the film thickness profile and the mean particle concentration. It has been used to discuss the self-pinning of receding contact lines that is related to the formation of rings of dried-in particles (coffeestain effect) that frequently occurs when films or drops of solutions or suspensions dewet by the combined effects of convection and evaporation.

One of the primary goals of researchers in this field, is the search for simple-to-use techniques that allow one to produce hierarchically structured functional layers for a wide range of applications such as, e.g., organic solar cells [98]. This means that the experiments advance very rapidly towards increasingly complex systems. For example, there have been investigations of the influence of the phase behaviour on the drying of droplets of a suspension of hard-sphere colloidal particles and non-adsorbing polymer [99], of the instabilities and the formation of drops in evaporating thin films of binary solutions [100] that may lead to treelike patterns [101], of effects of a secondary phase separation on evaporation-induced pattern formation in polymer films [102], and of the influence of an imposed flow on decomposition and deposition processes in a sliding ridge of evaporating solution of a binary polymer mixture [103] and of the influence of rather 
fast evaporation [104, 105]. These complex experimental systems all represent systems of high practical interest that the theories presented here are not (yet) able to describe. Such experiments do, however, provide a strong motivation for further work to extend the theories presented here, as well as to develop new approaches.

Let us finally mention that several topics were entirely excluded from our discussion here. First, we focused on a limited range of descriptions and did, for instance, not mention lattice Boltzmann, molecular dynamics or dissipative particle dynamics approaches that may also be employed to describe fluid suspensions [106-109]. Second, we have only discussed spatially homogeneous substrates. Patterned substrates are widely used in dewetting experiments [38, 110-112]. Theoretical descriptions are well developed for the dewetting of films of pure non-volatile liquids on such substrates [68, 113-119]. However, in the case of volatile liquids on heterogeneous substrates, much less work has been done. A third topic that we did not touch upon are possible continuum thin film approaches to demixing dewetting suspensions. We believe it is feasible to extend the diffuse interface theories such as model-H [120] to include the influence of evaporation in dewetting nanoparticle suspensions. For instance, such models have already been adapted to describe demixing free surface films of polymer blends [121- 123].

\section{Acknowledgments}

AJA and MJR gratefully acknowledge RCUK and EPSRC, respectively, for financial support. We acknowledge support by the European Union via the FP6 and FP7 Marie Curie schemes [Grants MRTN-CT-2004005728 (PATTERNS) and PITN-GA-2008-214919 (MULTIFLOW)].

[1] G. Reiter, "Dewetting of thin polymer films," Phys. Rev. Lett. 68, 75-78 (1992).

[2] G. Reiter, "Mobility of polymers in films thinner than their unperturbed size," Europhys. Lett. 23, $579-584$ (1993).

[3] A. Sharma and G. Reiter, "Instability of thin polymer films on coated substrates: Rupture, dewetting and drop formation," J. Colloid Interface Sci. 178, 383-399 (1996).

[4] P.-G. de Gennes, “Wetting: Statics and dynamics,” Rev. Mod. Phys. 57, 827-863 (1985). 
[5] F. Brochard-Wyart and J. Daillant, "Drying of solids wetted by thin liquid films," Can. J. Phys. 68, 1084-1088 (1989).

[6] P. Müller-Buschbaum, "Dewetting and pattern formation in thin polymer films as investigated in real and reciprocal space,” J. Phys.-Condes. Matter 15, R1549-R1582 (2003).

[7] R. Seemann, S. Herminghaus, C. Neto, S. Schlagowski, D. Podzimek, R. Konrad, H. Mantz, and K. Jacobs, "Dynamics and structure formation in thin polymer melt films," J. Phys.-Condes. Matter 17, S267-S290 (2005).

[8] U. Thiele, "Structure formation in thin liquid films," in S. Kalliadasis and U. Thiele, editors, "Thin films of Soft Matter," pages 25-93, Springer, Wien (2007).

[9] R. Xie, A. Karim, J. F. Douglas, C. C. Han, and R. A. Weiss, "Spinodal dewetting of thin polymer films," Phys. Rev. Lett. 81, 1251-1254 (1998).

[10] R. Seemann, S. Herminghaus, and K. Jacobs, "Dewetting patterns and molecular forces: A reconciliation,” Phys. Rev. Lett. 86, 5534-5537 (2001).

[11] U. Thiele, M. G. Velarde, and K. Neuffer, "Dewetting: Film rupture by nucleation in the spinodal regime," Phys. Rev. Lett. 87, 016104 (2001).

[12] M. Bestehorn and K. Neuffer, "Surface patterns of laterally extended thin liquid films in three dimensions,” Phys. Rev. Lett. 87, 046101 (2001).

[13] J. Becker, G. Grün, R. Seemann, H. Mantz, K. Jacobs, K. R. Mecke, and R. Blossey, “Complex dewetting scenarios captured by thin-film models," Nat. Mater. 2, 59-63 (2003).

[14] C. Redon, F. Brochard-Wyart, and F. Rondelez, "Dynamics of dewetting," Phys. Rev. Lett. 66, 715$718(1991)$

[15] R. Seemann, S. Herminghaus, and K. Jacobs, "Shape of a liquid front upon dewetting," Phys. Rev. Lett. 87, 196101 (2001).

[16] R. Fetzer, K. Jacobs, A. Münch, B. Wagner, and T. P. Witelski, "New slip regimes and the shape of dewetting thin liquid films," Phys. Rev. Lett. 95, 127801 (2005).

[17] F. Brochard-Wyart and C. Redon, "Dynamics of liquid rim instabilities," Langmuir 8, 2324-2329 (1992).

[18] G. Reiter and A. Sharma, "Auto-optimization of dewetting rates by rim instabilities in slipping polymer films," Phys. Rev. Lett. 87, 166103 (2001).

[19] A. Münch and B. Wagner, "Contact-line instability of dewetting thin films," Physica D 209, 178-190 (2005). 
[20] C. Tomlinson, “On the motion of certain liquids on the surface of water," Phil. Mag. Ser. 4 39, 32-48 (1870).

[21] C. G. Marangoni, "Ueber die Ausbreitung der Tropfen einer Flüssigkeit auf der Oberfläche einer anderen,” Ann. Phys. (Poggendorf) 143, 337-354 (1871).

[22] O. Karthaus, L. Grasjö, N. Maruyama, and M. Shimomura, "Formation of ordered mesoscopic polymer arrays by dewetting," Chaos 9, 308-314 (1999).

[23] X. Gu, D. Raghavan, J. F. Douglas, and A. Karim, "Hole-growth instability in the dewetting of evaporating polymer solution films,” J. Polym. Sci. Pt. B-Polym. Phys. 40, 2825-2832 (2002).

[24] S. W. Hong, J. F. Xia, and Z. Q. Lin, "Spontaneous formation of mesoscale polymer patterns in an evaporating bound solution," Adv. Mater. 19, 1413-1417 (2007).

[25] G. Liu, C. F. Zhang, J. Zhao, and Y. X. Zhu, "Study of the morphology of the three-phase contact line and its evolution by morphological examination after droplet evaporation of aqueous polymer solutions," Langmuir 24, 7923-7930 (2008).

[26] M. Mertig, U. Thiele, J. Bradt, G. Leibiger, W. Pompe, and H. Wendrock, "Scanning force microscopy and geometrical analysis of two-dimensional collagen network formation," Surface and Interface Analysis 25, 514-521 (1997).

[27] M. Mertig, U. Thiele, J. Bradt, D. Klemm, and W. Pompe, "Dewetting of thin collagenous precursor films,” Appl. Phys. A 66, S565-S568 (1998).

[28] U. Thiele, M. Mertig, and W. Pompe, "Dewetting of an evaporating thin liquid film: Heterogeneous nucleation and surface instability," Phys. Rev. Lett. 80, 2869-2872 (1998).

[29] H. Maeda, "An atomic force microscopy study of ordered molecular assemblies and concentric ring patterns from evaporating droplets of collagen solutions," Langmuir 15, 8505-8513 (1999).

[30] I. I. Smalyukh, O. V. Zribi, J. C. Butler, O. D. Lavrentovich, and G. C. L. Wong, "Structure and dynamics of liquid crystalline pattern formation in drying droplets of DNA," Phys. Rev. Lett. 96, 177801 (2006).

[31] L. Zhang, S. Maheshwari, H. C. Chang, and Y. X. Zhu, "Evaporative self-assembly from complex DNA-colloid suspensions," Langmuir 24, 3911-3917 (2008).

[32] M. Maillard, L. Motte, A. T. Ngo, and M. P. Pileni, "Rings and hexagons made of nanocrystals: A Marangoni effect," J. Phys. Chem. B 104, 11871-11877 (2000).

[33] G. L. Ge and L. Brus, "Evidence for spinodal phase separation in two-dimensional nanocrystal selfassembly,” J. Phys. Chem. B 104, 9573-9575 (2000). 
[34] P. Moriarty, M. D. R. Taylor, and M. Brust, “Nanostructured cellular networks,” Phys. Rev. Lett. 89, 248303 (2002).

[35] E. Rabani, D. R. Reichman, P. L. Geissler, and L. E. Brus, "Drying-mediated self-assembly of nanoparticles," Nature 426, 271-274 (2003).

[36] L. V. Govor, G. Reiter, J. Parisi, and G. H. Bauer, "Self-assembled nanoparticle deposits formed at the contact line of evaporating micrometer-size droplets," Phys. Rev. E 69, 061609 (2004).

[37] C. P. Martin, M. O. Blunt, and P. Moriarty, "Nanoparticle networks on silicon: Self-organized or disorganized?" Nano Lett. 4, 2389-2392 (2004).

[38] C. P. Martin, M. O. Blunt, E. Pauliac-Vaujour, A. Stannard, P. Moriarty, I. Vancea, and U. Thiele, "Controlling pattern formation in nanoparticle assemblies via directed solvent dewetting," Phys. Rev. Lett. 99, 116103 (2007).

[39] A. Stannard, C. P. Martin, E. Pauliac-Vaujour, P. Moriarty, and U. Thiele, "Dual-scale pattern formation in nanoparticle assemblies," J. Chem. Phys. C 112, 15195-15203 (2008).

[40] E. Pauliac-Vaujour, A. Stannard, C. P. Martin, M. O. Blunt, I. Notingher, P. J. Moriarty, I. Vancea, and U. Thiele, "Fingering instabilities in dewetting nanofluids," Phys. Rev. Lett. 100, 176102 (2008).

[41] I. Vancea, U. Thiele, E. Pauliac-Vaujour, A. Stannard, C. P. Martin, M. O. Blunt, and P. J. Moriarty, “Front instabilities in evaporatively dewetting nanofluids," Phys. Rev. E 78, 041601 (2008).

[42] U. Thiele, Entnetzung von Kollagenfilmen, Ph.D. thesis, Technische Universität Dresden (1998).

[43] H. Yabu and M. Shimomura, "Preparation of self-organized mesoscale polymer patterns on a solid substrate: Continuous pattern formation from a receding meniscus,” Adv. Funct. Mater. 15, 575-581 (2005).

[44] R. D. Deegan, O. Bakajin, T. F. Dupont, G. Huber, S. R. Nagel, and T. A. Witten, "Capillary flow as the cause of ring stains from dried liquid drops," Nature 389, 827-829 (1997).

[45] E. Adachi, A. S. Dimitrov, and K. Nagayama, "Stripe patterns formed on a glass-surface during droplet evaporation,” Langmuir 11, 1057-1060 (1995).

[46] R. D. Deegan, “Pattern formation in drying drops,” Phys. Rev. E 61, 475-485 (2000).

[47] R. D. Deegan, O. Bakajin, T. F. Dupont, G. Huber, S. R. Nagel, and T. A. Witten, "Contact line deposits in an evaporating drop," Phys. Rev. E 62, 756-765 (2000).

[48] L. Shmuylovich, A. Q. Shen, and H. A. Stone, "Surface morphology of drying latex films: Multiple ring formation," Langmuir 18, 3441-3445 (2002).

[49] V. X. Nguyen and K. J. Stebe, "Patterning of small particles by a surfactant-enhanced Marangoni- 
Benard instability,” Phys. Rev. Lett. 88, 164501 (2002).

[50] J. Huang, F. Kim, A. R. Tao, S. Connor, and P. Yang, "Spontaneous formation of nanoparticle stripe patterns through dewetting," Nat. Mater. 4, 896-900 (2005).

[51] S. H. Lee, P. J. Yoo, S. J. Kwon, and H. H. Lee, “Solvent-driven dewetting and rim instability,” J. Chem. Phys. 121, 4346-4351 (2004).

[52] L. Xu, T. F. Shi, P. K. Dutta, and L. An, "Rim instability by solvent-induced dewetting," J. Chem. Phys. 127, 144704 (2007).

[53] L. Xu, T. F. Shi, and L. J. An, "The dewetting dynamics of the polymer thin film by solvent annealing,” J. Chem. Phys. 129, 044904 (2008).

[54] M. Elbaum and S. G. Lipson, "How does a thin wetted film dry up?" Phys. Rev. Lett. 72, 3562-3565 (1994).

[55] N. Samid-Merzel, S. G. Lipson, and D. S. Tannhauser, "Pattern formation in drying water films," Phys. Rev. E 57, 2906-2913 (1998).

[56] A. Padmakar, K. Kargupta, and A. Sharma, "Instability and dewetting of evaporating thin water films on partially and completely wettable substrates,” J. Chem. Phys. 110, 1735-1744 (1999).

[57] A. V. Lyushnin, A. A. Golovin, and L. M. Pismen, "Fingering instability of thin evaporating liquid films," Phys. Rev. E 65, 021602 (2002).

[58] L. M. Pismen, “Spinodal dewetting in a volatile liquid film,” Phys. Rev. E 70, 021601 (2004).

[59] C. Poulard, O. Benichou, and A. M. Cazabat, "Freely receding evaporating droplets," Langmuir 19, 8828-8834 (2003).

[60] Y. Gotkis, I. Ivanov, N. Murisic, and L. Kondic, "Dynamic structure formation at the fronts of volatile liquid drops,” Phys. Rev. Lett. 97, 186101 (2006).

[61] E. Pauliac-Vaujour and P. Moriarty, "Meniscus-mediated organization of colloidal nanoparticles," J. Phys. Chem. C 111, 16255-16260 (2007).

[62] C. Gigault, K. Dalnoki-Veress, and J. R. Dutcher, "Changes in the morphology of self-assembled polystyrene microsphere monolayers produced by annealing," J. Colloid Interface Sci. 243, 143-155 (2001).

[63] A. Oron, S. H. Davis, and S. G. Bankoff, "Long-scale evolution of thin liquid films," Rev. Mod. Phys. 69, 931-980 (1997).

[64] U. Thiele, "Thin film evolution equations from (evaporating) dewetting liquid layers to epitaxial growth,” J. Phys.-Cond. Mat. (2010), (at press). 
[65] J. P. Burelbach, S. G. Bankoff, and S. H. Davis, "Nonlinear stability of evaporating/condensing liquid films," J. Fluid Mech. 195, 463-494 (1988).

[66] A. Oron and S. G. Bankoff, "Dewetting of a heated surface by an evaporating liquid film under conjoining/disjoining pressures," J. Colloid Interface Sci. 218, 152-166 (1999).

[67] L. W. Schwartz, R. V. Roy, R. R. Eley, and S. Petrash, "Dewetting patterns in a drying liquid film," J. Colloid Interface Sci. 214, 363-374 (2001).

[68] K. Kargupta, R. Konnur, and A. Sharma, "Spontaneous dewetting and ordered patterns in evaporating thin liquid films on homogeneous and heterogeneous substrates," Langmuir 17, 1294-1305 (2001).

[69] M. Bestehorn and D. Merkt, "Regular surface patterns on Rayleigh-Taylor unstable evaporating films heated from below," Phys. Rev. Lett. 97, 127802 (2006).

[70] G. F. Teletzke, H. T. Davis, and L. E. Scriven, “Wetting hydrodynamics,” Rev. Phys. Appl. 23, 9891007 (1988).

[71] J. N. Israelachvili, Intermolecular and Surface Forces, Academic Press, London (1992).

[72] V. S. Mitlin, "Dewetting of solid surface: Analogy with spinodal decomposition," J. Colloid Interface Sci. 156, 491-497 (1993).

[73] L. M. Pismen and Y. Pomeau, "Disjoining potential and spreading of thin liquid layers in the diffuse interface model coupled to hydrodynamics," Phys. Rev. E 62, 2480-2492 (2000).

[74] L. Onsager, "Crystal statistics. I. A two-dimensional model with an order-disorder transition," Phys. Rev. 65, 117-149 (1944).

[75] G. Reiter, “Unstable thin polymer films: Rupture and dewetting processes," Langmuir 9, 1344-1351 (1993).

[76] C. G. Sztrum, O. Hod, and E. Rabani, "Self-assembly of nanoparticles in three-dimensions: Formation of stalagmites," J. Phys. Chem. B 109, 6741-6747 (2005).

[77] G. Yosef and E. Rabani, "Self-assembly of nanoparticles into rings: A lattice-gas model," J. Phys. Chem. B 110, 20965-20972 (2006).

[78] J. F. Gouyet, M. Plapp, W. Dieterich, and P. Maass, "Description of far-from-equilibrium processes by mean-field lattice gas models," Adv. Phys. 52, 523-638 (2003).

[79] U. M. B. Marconi and P. Tarazona, "Dynamic density functional theory of fluids," J. Chem. Phys. 110, 8032-8044 (1999).

[80] U. M. B. Marconi and P. Tarazona, "Dynamic density functional theory of fluids," J. Phys.-Condes. Matter 12, A413-A418 (2000). 
[81] A. J. Archer and M. Rauscher, "Dynamical density functional theory for interacting brownian particles: Stochastic or deterministic?” J. Phys. A-Math. Gen. 37, 9325-9333 (2004).

[82] A. J. Archer and R. Evans, "Dynamical density functional theory and its application to spinodal decomposition,” J. Chem. Phys. 121, 4246-4254 (2004).

[83] P. A. Monson, "Mean field kinetic theory for a lattice gas model of fluids confined in porous materials,” J. Chem. Phys. 128, 084701 (2008).

[84] P. M. Chaikin and T. C. Lubensky, Principles of condensed matter physics, Cambridge University Press (1997).

[85] J. S. Langer, "An introduction to the kinetics of first-order phase transitions," in C. Godreche, editor, “Solids far from Equilibrium,” pages 297-363, Cambridge University Press (1992).

[86] M. A. Spaid and G. M. Homsy, "Stability of Newtonian and viscoelastic dynamic contact lines," Phys. Fluids 8, 460-478 (1996).

[87] U. Thiele and E. Knobloch, "Front and back instability of a liquid film on a slightly inclined plate," Phys. Fluids 15, 892-907 (2003).

[88] M. R. E. Warner, R. V. Craster, and O. K. Matar, "Surface patterning via evaporation of ultrathin films containing nanoparticles," J. Colloid Interface Sci. 267, 92-110 (2003).

[89] O. K. Matar, R. V. Craster, and K. Sefiane, "Dynamic spreading of droplets containing nanoparticles," Phys. Rev. E 76, 056315 (2007).

[90] J. J. Zhou, B. Dupuy, A. L. Bertozzi, and A. E. Hosoi, "Theory for shock dynamics in particle-laden thin films," Phys. Rev. Lett. 94, 117803 (2005).

[91] B. P. Cook, A. L. Bertozzi, and A. E. Hosoi, "Shock solutions for particle-laden thin films," SIAM J. Appl. Math. 68, 760-783 (2008).

[92] R. V. Craster, O. K. Matar, and K. Sefiane, "Pinning, retraction, and terracing of evaporating droplets containing nanoparticles," Langmuir (2009), online available.

[93] D. Quemada, "Rheology of concentrated disperse systems and minimum energy-dissipation principle I. Viscosity-concentration relationship,” Rheol. Acta 16, 82-94 (1977).

[94] D. Quemada and C. Berli, "Energy of interaction in colloids and its implications in rheological modeling," Adv. Colloid Interface Sci. 98, 51-85 (2002).

[95] J. J. Stickel and R. L. Powell, "Fluid mechanics and rheology of dense suspensions," Annu. Rev. Fluid Mech. 37, 129-149 (2005).

[96] J. K. G. Dhont, An Introduction to Dynamics of Colloids, Elsevier, Amsterdam (1996). 
[97] U. Thiele, M. G. Velarde, K. Neuffer, and Y. Pomeau, "Film rupture in the diffuse interface model coupled to hydrodynamics," Phys. Rev. E 64, 031602 (2001).

[98] J. Heier, J. Groenewold, F. A. Castro, F. Nueesch, and R. Hany, "Enlarged bilayer interfaces from liquid-liquid dewetting for photovoltaic applications,” P Soc Photo-Opt Instrum Eng 6999, J9991J9991 (2008).

[99] M. D. Haw, M. Gillie, and W. C. K. Poon, "Effects of phase behavior on the drying of colloidal suspensions," Langmuir 18, 1626-1633 (2002).

[100] L. V. Govor, J. Parisi, G. H. Bauer, and G. Reiter, "Instability and droplet formation in evaporating thin films of a binary solution,” Phys. Rev. E 71, 051603 (2005).

[101] L. V. Govor, G. Reiter, G. H. Bauer, and J. Parisi, "Self-assembled treelike patterns from an evaporating binary solution,” Phys. Rev. E 74, 061603 (2006).

[102] M. Yamamura, T. Nishio, T. Kajiwara, and K. Adachi, "Evaporation-induced pattern formation in polymer films via secondary phase separation,” Chem. Eng. Sci. 57, 2901-2905 (2002).

[103] P. Müller-Buschbaum, E. Bauer, S. Pfister, S. V. Roth, M. Burghammer, C. Riekel, C. David, and U. Thiele, "Creation of multi-scale stripe-like patterns in thin polymer blend films," Europhys. Lett. 73, 35-41 (2006).

[104] E. Bormashenko, R. Pogreb, O. Stanevsky, Y. Bormashenko, T. Stein, and O. Gengelman, "Mesoscopic patterning in evaporated polymer solutions: New experimental data and physical mechanisms," Langmuir 21, 9604-9609 (2005).

[105] E. Bormashenko, R. Pogreb, O. Stanevsky, Y. Bormashenko, T. Stein, V. Z. Gaisin, R. Cohen, and O. V. Gendelman, "Mesoscopic patterning in thin polymer films formed under the fast dip-coating process," Macromol. Mater. Eng. 290, 114-121 (2005).

[106] J. B. Gibson, K. Zhang, K. Chen, S. Chynoweth, and C. W. Manke, "Simulation of colloid-polymer systems using dissipative particle dynamics," Mol. Simul. 23, 1-41 (1999).

[107] K. Stratford and I. Pagonabarraga, "Parallel simulation of particle suspensions with the lattice Boltzmann method," Comput. Math. Appl. 55, 1585-1593 (2008).

[108] G. Drazer, B. Khusid, J. Koplik, and A. Acrivos, "Wetting and particle adsorption in nanoflows," Phys. Fluids 17, 017102 (2005).

[109] J. Kromkamp, D. van den Ende, D. Kandhai, R. van der Sman, and R. Boom, "Lattice Boltzmann simulation of $2 \mathrm{~d}$ and $3 \mathrm{~d}$ non-Brownian suspensions in Couette flow," Chem. Eng. Sci. 61, 858-873 (2006). 
[110] L. Rockford, Y. Liu, P. Mansky, T. P. Russell, M. Yoon, and S. G. J. Mochrie, "Polymers on nanoperiodic, heterogeneous surfaces," Phys. Rev. Lett. 82, 2602-2605 (1999).

[111] A. Sehgal, V. Ferreiro, J. F. Douglas, E. J. Amis, and A. Karim, "Pattern-directed dewetting of ultrathin polymer films," Langmuir 18, 7041-7048 (2002).

[112] M. Geoghegan and G. Krausch, "Wetting at polymer surfaces and interfaces," Prog. Polym. Sci. 28, 261-302 (2003).

[113] P. Lenz and R. Lipowsky, "Morphological transitions of wetting layers on structured surfaces," Phys. Rev. Lett. 80, 1920-1923 (1998).

[114] C. Bauer, S. Dietrich, and A. O. Parry, "Morphological phase transitions of thin fluid films on chemically structured substrates," Europhys. Lett. 47, 474-480 (1999).

[115] R. Konnur, K. Kargupta, and A. Sharma, "Instability and morphology of thin liquid films on chemically heterogeneous substrates," Phys. Rev. Lett. 84, 931-934 (2000).

[116] M. Brinkmann and R. Lipowsky, "Wetting morphologies on substrates with striped surface domains," J. Appl. Phys. 92, 4296-4306 (2002).

[117] L. Brusch, H. Kühne, U. Thiele, and M. Bär, "Dewetting of thin films on heterogeneous substrates: Pinning vs. coarsening," Phys. Rev. E 66, 011602 (2002).

[118] U. Thiele, L. Brusch, M. Bestehorn, and M. Bär, "Modelling thin-film dewetting on structured substrates and templates: Bifurcation analysis and numerical simulations,” Eur. Phys. J. E 11, 255-271 (2003).

[119] U. Thiele, "Open questions and promising new fields in dewetting," Eur. Phys. J. E 12, 409-416 (2003).

[120] D. M. Anderson, G. B. McFadden, and A. A. Wheeler, "Diffuse-interface methods in fluid mechanics,” Ann. Rev. Fluid Mech. 30, 139-165 (1998).

[121] U. Thiele, S. Madruga, and L. Frastia, "Decomposition driven interface evolution for layers of binary mixtures: I. Model derivation and stratified base states,” Phys. Fluids 19, 122106 (2007).

[122] O. A. Frolovskaya, A. A. Nepomnyashchy, A. Oron, and A. A. Golovin, "Stability of a two-layer binary-fluid system with a diffuse interface,” Phys. Fluids 20, 112105 (2008).

[123] S. Madruga and U. Thiele, "Decomposition driven interface evolution for layers of binary mixtures: II. Influence of convective transport on linear stability,” Phys. Fluids 21, 062104 (2009). 